\title{
NAVIGATIONAL COMPASS IN MAGNETIC BACTERIA
}

\author{
R. B. FRANKEL \\ Francls Bitter National Magnet Laboratory, MIT, Cambridge, MA 02319, USA \\ and R. P. BLAKEMORE \\ Depr. of Microbiology, University of New Hampshire, Durham, NH 09824, USA
}

\begin{abstract}
Magnetotactic bacteria that orient and swim in a preferred direction in the geomagnetic field contain sufficient single domain magnetite to constitute a biomagnetic compass.
\end{abstract}

Several species of bacteria from both fresh water and marine enviornments were observed to orient and swim in a preferred direction in the geomagnetic field (magnetotaxis) [1, 2]. Kalmijn and Blakemore [2] observed that most of the magnetotactic bacteria in sediments oriented and swam along magnetic field lines toward the north. Reversal of the ambient magnetic field with Helmholtz coils caused the cells to make large U-turns within one second and swim in the opposite direction. Killed cells also oriented in uniform fields as low as $0.1 \mathrm{G}$. In these and other respects [3] the cells behaved like single magnetic dipoles.

A magnetotactic spirillum designated strain MS-1 was isolated from a fresh water swamp and grown in pure culture [4]. Magnetic cells of this new bacterial isolate each had an intracytoplasmic chain of approximately 22 electron opaque particles (fig. 1). Each particle was roughly octahedral, $500 \AA$ along each major axis. Energy dispersive $\mathrm{X}$-ray measurements [1] (and unpublished results) showed that the particles in strain MS-1 had high iron content. Mössbauer spectroscopy of ${ }^{57} \mathrm{Fe}$ in freeze dried magnetic and nonmagnetic cells [5] showed conclusively that iron in magnetic cells was present primarily in the form of magnetite, $\mathrm{Fe}_{3} \mathrm{O}_{4}$, with a small admixture of $\gamma-\mathrm{Fe}_{2} \mathrm{O}_{3}$. Another ironcontaining material with a room temperature Mössbauer spectrum similar to that of the iron storage protein ferritin was also observed.

A population of nonmagnetic cells of MS-1 was derived by successively cloning their nonmagnetotactic progeny in media low in iron. The magnetic variant of MS-1 had over 10 times the total iron content of the nonmagnetic form. Nonmagnetotactic cells lacked the magnetite crystals. Thus, magnetotaxis in MS-1 is associated with intracellular magnetite. Since the bacteria were grown in a chemically defined media containing soluble (chelated) iron, the presence of intracellular magnetite implies a process of bacterial synthesis.

The magnetic properties of magnetite particles depend on their size and shape. For a particle of roughly cubic shape with side dimension $d$, there is a range of $d$ over which the particle will be a single
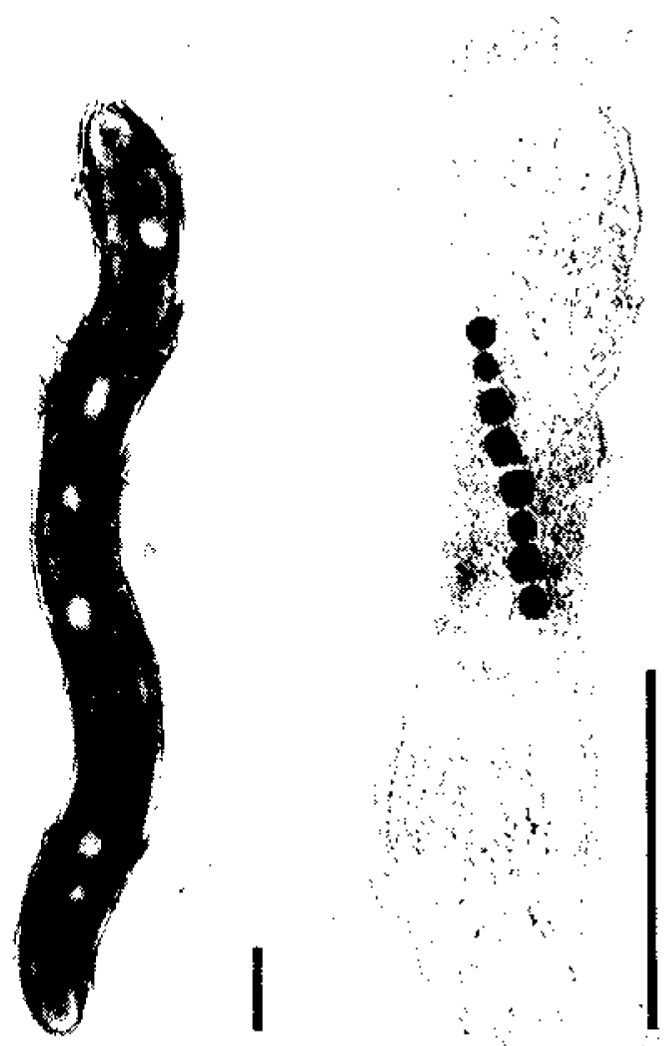

Fig. 1. Electron micrographs of magnetotactic spirillum strain MS-1, Left: cell stained with phosphotungstic acid. Flagella have been displaced from normal positions at ends of cell. Note chain of magnetite particles. Right: cell thin-sectioned to reveal internal location of chain of magnetite particles. Each bar $=0.5$ $\mu \mathrm{m}$. 
magnetic domain. The magnetic moment of a single domain for temperatures well below the Curie point is the saturation or maximum magnetic moment of the particle and is unchanging in time. Particles with dimensions $d \leqslant d_{\mathrm{s}}$ are superparamagnetic, i.e. thermal activation induces transitions of the magnetic moment between different easy magnetic axis directions with a consequent loss of magnetic "memory" [6]. Particles with dimensions $d>d_{\mathrm{m}}$ are multidomain and consequently have macroscopic moments which are either nulled or less per unit volume than single domain particles. Butler and Banerjee [7] calculated that for magnetite at $300 \mathrm{~K} d_{\mathrm{s}} \approx 500 \AA$ and $d_{\mathrm{m}} \approx 760 \AA$. Thus with $d=500 \AA$, the magnetite particles in strain MS-1 are within the single domain size range.

The magnetic properties of a chain of single domain particles have been considered by Jacobs and Bean [8]. Their results showed that because of strong interparticle interactions, the preferred orientation of the individual particles is such that their axes of magnetization are parallel, north-tosouth along the chain direction. Thus, the entire chain acts as a single magnetic dipole with a moment equal to the sum of the particle moments. They also calculated the applied field necessary to cause reversal of the chain magnetization, postulating a fanning mechanism for individual moment reversal. This mechanism requires an applied field of several hundred Gauss to induce reversal in a chain of magnetite particles, in qualitative agreement with recent experimental results obtained using magnetic bacteria [3].

The magnetic moment per bacterium, $M$, can be calculated using the known magnetic moment per unit volume of magnetite, $M_{\mathrm{v}}=480 \mathrm{emu} / \mathrm{cm}^{3}$ [9]. For a single particle of volume $1.25 \times 10^{-16} \mathrm{~cm}^{3}$, the magnetic moment $m=6.1 \times 10^{-14} \mathrm{emu}$. For a cell containing the average chain length of 22 particles, the total moment per bacterium $M=1.3 \times$ $10^{-12}$ emu. In the geomagnetic field of $0.5 \mathrm{G}$, the total magnetic energy of a cell $M H=6.6 \times 10^{-13}$ erg. This value is over an order of magnitude greater than thermal energy, $k T\left(4.1 \times 10^{-14}\right.$ erg at $300 \mathrm{~K}$ ).

The orientation of a bacterium or, equivalently, the orientation of an ensemble of bacteria in the earth's magnetic field of $0.5 \mathrm{G}$ in water at ambient temperatures, can be calculated from the well- known Langevin theory of classical paramagnetism [10]. The energy of a magnetic dipole in a magnetic field $E=M \cdot H=M H \cos \theta$, where $\theta$ is the angle between the moment direction and the magnetic field direction. The orientation is equivalent to the thermal average of $\cos \theta$, which is a function of $M H / k T$ :

$$
\langle\cos \theta\rangle=L(M H / k T),
$$

where

$$
\mathrm{L}\left(\frac{M H}{k T}\right)=\operatorname{ctnh}\left(\frac{M H}{k T}\right)-\left(\frac{M H}{k T}\right)^{-1} .
$$

This function is plotted in fig. 2. The arrow indicates the orientation of a magnetic bacterium with a chain of 22 particles. This shows that each bacterium contains a sufficient but not an excessive amount of single domain sized magnetite in an appropriate configuration to produce orientation in the earth's magnetic field at ambient temperature, e.g. the cell's chain of magnetite crystals functions as a biomagnetic compass.

The simplest hypothesis for the mechanism of magnetotaxis is passive orientation of the bacterium resulting from the torque exerted by the ambient magnetic field on its biomagnetic compass

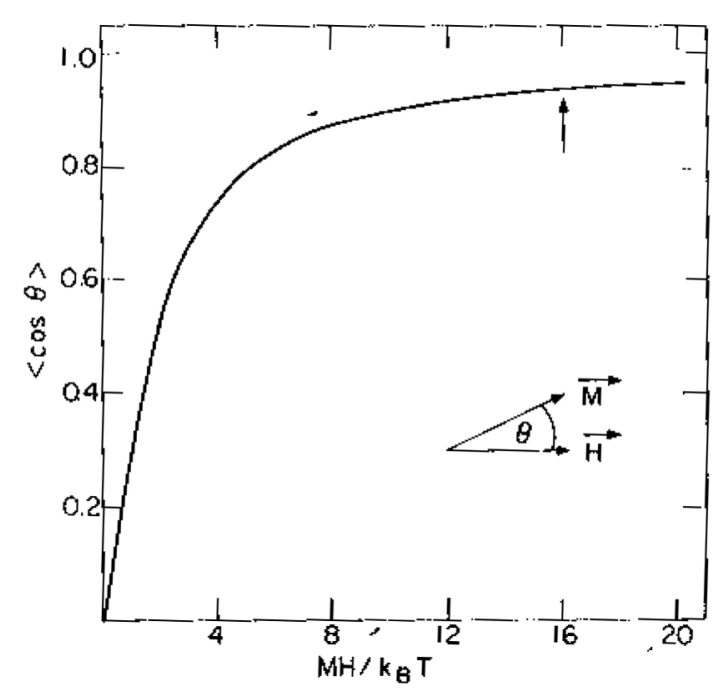

Fig. 2. Langevin function (eq. (1) plotted as a function of the ratio of magnetic to thermal energy. The arrow indicates the orientation of magnetic bacteria with a calculated moment of $1.3 \times 10^{-12} \mathrm{emu} /$ cell in the geomagnetic field at ambient temperature. 
as it swims. An estimate of the rotation time required for a bacterium in water can be obtained from the rotational diffusion equation [10-12]. As discussed by Purcell [13], the motion of a bacterium in water is dominated by viscous forces, and hence the torque is proportional to the angular velocity of rotation:

$$
M \times H=8 \pi r^{3} \eta \mathrm{d} \theta / \mathrm{d} t,
$$

where $\eta$ is the viscosity of water $(0.01$ poise at 300 $\mathrm{K})$ and $r$ is the radius of the bacterium, using a spherical approximation. We define an orientation time constant

$$
\tau=8 \pi \eta r^{3} / M H \approx 0.05 \mathrm{~s}
$$

assuming $r=0.5 \mu \mathrm{m}$ and $M H$ as given above. The time required to reorient $180^{\circ}$ in response to a sign reversal of an $0.5 \mathrm{G}$ magnetic field is $\sim 8 \tau$ or $0.4 \mathrm{~s}$, This result is of the order of reversal times for bacteria from mud samples [2].

Since most magnetotactic bacteria from the northern hemisphere are observed to swim northward and hence downward [1,14], the compass in these cells must have a fixed orientation with respect to the flagellum, with the north-seeking pole opposite to the flagellum. This orientation could be preserved in cell division if the compass is partitioned between two daughter cells. Subsequently, during the magnetite biosynthesis the magnetic moments of nascent magnetite particles at the ends of the pre-existing chains would become oriented along the chain direction by interaction with the chain dipole moment. It is also interesting to speculate that if magnetotactic bacteria exist in the southern hemisphere, and if downward-directed motion is advantageous, they might have reversed polarity, i.e. swim in the direction of their southseeking pole [15]. South-seeking bacteria have been produced in the laboratory by subjecting them to short (1 $\mu \mathrm{s}$ ), powerful (up to $600 \mathrm{G}$ ) magnetic pulses oriented opposite to their swimming direction [3].

In conclusion, magnetotactic bacteria construct a biomagnetic compass by synthesizing a chain of single domain magnetite particles. This chain functions as a single magnetic dipole with a magnetic moment sufficient to produce orientation of the bacterium in the geomagnetic field at ambient temperatures. There are at least two consequences of a biomagnetic compass in bacteria: (i) they will align in, and their motion will be directed in, the (homogeneous) geomagnetic field; (ii) they will tend to localize by swimming to regions of locally higher magnetic flux density due, for example, to materials with high magnetic susceptibility in the environment.

We are pleased to acknowledge D. L. Balkwill and $D$. Maratea for electron microscopy. We are also grateful to E. M. Purcell and C. P. Bean for private communications and encouragement, and to Ad. J. Kalmijn for continuing in terest and collaboration. The Francis Bitter National Magnet Laboratory is supported by the National Science Foundation. RPB acknowledges support by NSF grant PCM77-12175. Work partially supported by the Office of Naval Research.

\section{References}

[1] R. P. Blakemore, Science 190 (1975) 377.

[2] Ad. J. Kalmijn and R. P. Blakemore, Proc. Intern. Union Physiol. Sci. 13 (1977) 364.

[3] Ad. J. Kalmijn and R. P. Blakemore, in: eds. K. SchmidtKoenig and W. T. Keeton, Animal Migration, Navigation and Homing (Springer Verlag, New York, 1978) p. 344.

[4] R. P. Blakemore, D. Maratea and R. S. Wolfe, J. Bacteriol., in press.

[5] R. B. Frankel, R. P. Blakemore and R. S. Wolfe, Science 203 (1979) 1355.

[6] C. P. Bean and J. D. Livingston, J. Appl. Phys. 30 (1959) $120 \mathrm{~S}$.

[7] R. F. Butler and S. K. Banerjee, J. Geophys. Res. 80 (1975) 4049.

[8] I. S. Jacobs and C. P. Bean, Phys. Rev. 100 (1955) 1060.

[9] American Institute of Physics Handbook (McGraw-Hill, New York, 1957).

[10] Also noted independently by C. P. Bean (private communication).

[11] Also noted independently by E. M. Purcell (private conmunication).

[12] E. D. Yorke, J. Theol. Biology 77 (1979) 101.

[13] E. M. Purcell, Am. J. Phys. 45 (1977) 3.

[14] T, T. Moensch and W. A. Konetzka, Arch. Microbiol. 119 (1978) 203.

[15] R. P. Blakemore, Ad. J. Kalmijn and R. B. Frankel, unpublished. 